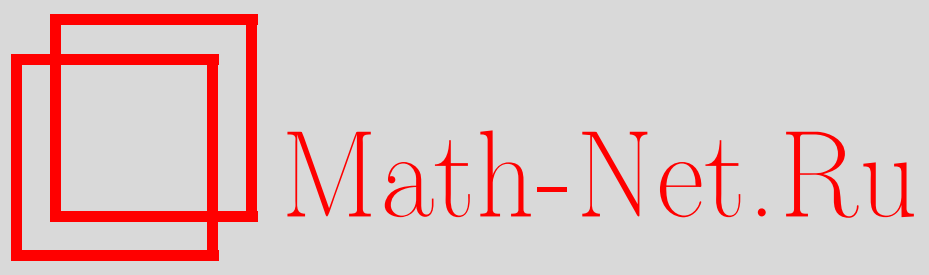

И. Я. Арефьева, Р. В. Горбачев, П. Б. Медведев, Д. В. Рычков, Соотношения спуска и осцилляторный метод обрезания по уровням, ТMФ, 2007, том 150, номер 1, 5-25

DOI: https://doi.org/10.4213/tmf5962

Использование Общероссийского математического портала Math-Net.Ru подразумевает, что вы прочитали и согласны с пользовательским соглашением http://www . mathnet.ru/rus/agreement

Параметры загрузки:

IP : 52.205 .19 .152

26 апреля 2023 г., 16:01:18

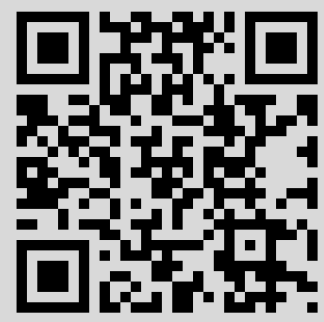




\title{
СООТНОШЕНИЯ СПУСКА И ОСЦИЛЛЯТОРНЫЙ МЕТОД ОБРЕЗАНИЯ ПО УРОВНЯМ
}

\begin{abstract}
Проверяется осцилляторный метод обрезания по уровням в бозонной струнной теории поля с помощью вычисления соотношения спуска $\left\langle V_{3} \mid V_{1}\right\rangle=\widetilde{Z}_{3}\left\langle V_{2}\right|$. Для сектора духов используются фермионные вершины в стандартном осцилляторном базисе. Предлагаются две схемы вычисления: в первой используется условие оверлэпа по отношению к вставкам; во второй производятся прямые вычисления. В обеих схемах получены правильные структуры показателя экспоненты и предэкспоненциального фактора вершины $\left\langle V_{2}\right|$, однако появляются различные нормировочные факторы $\widetilde{Z}_{3}$.
\end{abstract}

Ключевые слова: струнная теория поля, обрезание по уровням.

\section{1. ВВЕДЕНИЕ}

Кубическая бозонная струнная теория поля (КБСТП) Виттена [1] имеет замечательные алгебраические свойства. Поля в этой теории принадлежат бесконечномерной некоммутативной $\mathbb{Z}_{2}$-градуированной ассоциативной алгебре. Действие имеет вид действия Черна-Саймонса

$$
S=\frac{1}{2} \int \Phi \star Q \Phi+\frac{g}{3} \int \Phi \star \Phi \star \Phi .
$$

Представления виттеновской алгебры построены как на осцилляторном, так и на конформном языках [2], [3] (см. обзоры [4], [5]).

Чтобы сформулировать КБСТП в осцилляторном представлении, достаточно сконструировать только две вершины: $\left\langle\left|V_{3}\right|\right\rangle$, которая представляет, и $\left\langle V_{1}\right|$, которая представляет $\int[2]$. В таком представлении струнные поля $\Phi$ реализуются как кет-векторы $|\Phi\rangle$ в фоковском пространстве. Вершины $V_{1}, V_{3}$ определяются с

* Математический институт им. В. А. Стеклова РАН, Москва, Россия.

E-mail: arefeva@mi.ras.ru,rgorbachev@mi.ras.ru

${ }^{\dagger}$ Институт теоретической и экспериментальной физики, Москва, Россия. E-mail: pmedvedev@itep.ru

${ }^{\ddagger}$ Московский государственный университет, Москва, Россия. E-mail: rogdin@gmail.com 
точностью до нормировочных факторов, которые могут быть удалены переопределением заряда и струнного поля.

Основная трудность в вычислениях с вершинами $V_{i}$ - то, что они определяются бесконечномерными матрицами [2], [6]-[8]. Для того чтобы провести вычисления, можно использовать конечное приближение этих матриц [9]. Этот метод называется осцилляторным методом обрезания по уровням.

Альтернативным является метод обрезания по полям, который был предложен в работах [10], [11] и с большим успехом применен в работах [12] для вычисления тахионного потенциала. Член четвертого порядка в тахионном потенциале может быть также получен из борновской 4-точечной КБСТП-диаграммы при нулевом импульсе [9], [10] методом осцилляторного обрезания по уровням. Оказалось [9], что этот результат находится в хорошем согласии с тахионным потенциалом, полученным с помощью метода обрезания по полям.

Другой проверкой осцилляторного метода обрезания по уровням может являться вычисление некоторых точных соотношений. В работе [13] была предложена проверка соотношения спуска между струнными вершинами. Соотношения спуска [2] устанавливают естественную связь между $\mathrm{N}$-струнными вершинами, $N \geqslant 1$ :

$$
\left\langle V_{N+1} \mid V_{1}\right\rangle=\left\langle V_{N}\right|
$$

В работах [13], [14] было найдено, что $\left\langle V_{3} \mid V_{1}\right\rangle=\widetilde{Z}_{3}\left\langle V_{2}\right|$, где $\widetilde{Z}_{3} \neq 1$; появление $\widetilde{Z}_{N} \neq 1$ в соотношениях спуска было предложено рассматривать как аномалию. Мы обсудим эту интерпретацию в разделе 2. Для того чтобы понять происхождение $\widetilde{Z}_{N} \neq 1$, нужно более детально исследовать соотношения спуска вершин. В действительности нельзя априори ожидать, что $\widetilde{Z}_{N}=1$ для произвольных вершин $V_{1}, V_{N}$ и $V_{N+1}$. Мы предлагаем самосогласованную схему определения вершин $\left\langle\widehat{V}_{N}\right|, N \geqslant 2$, с помощью $\left\langle V_{1}\right|$ и $\left\langle\left|V_{3}\right|\right\rangle$, которые удовлетворяют соотношению (1.2). Для вершин $\left\langle V_{N}\right|$, полученных как решения уравнений оверлэпа и использующихся в реальных вычислениях, нельзя ожидать выполнения соотношения (1.2), так как решения этих уравнений определяются с точностью до множителя. Вообще говоря, вместо уравнения (1.2) мы имеем соотношение спуска в виде

$$
\left\langle V_{N+1} \mid V_{1}\right\rangle=Z_{N} Z_{-1}^{-1} Z_{N+1}^{-1}\left\langle V_{N}\right|
$$

Такая факторизация была обнаружена в работе [15] как результат прямого вычисления с вершинами в непрерывном $\kappa$-базисе [16]. Заметим, что иногда более удобно аналитически проводить вычисления, когда матрицы представлены в диагональной форме в непрерывном $\kappa$-базисе [14].

В разделе 3 мы проверяем соотношение спуска для вершин $\left\langle V_{2}\right|$ и $\left\langle V_{3}\right|$, полученных как решения уравнений оверлэпа. В первом случае вычисления аналогичны тем, которые были проведены в работах [2], и дают $\widetilde{Z}_{2}=1$ без применения метода обрезания. Во втором случае в секторе духов мы используем две схемы: одну (обозначенную как (1)) с использованием уравнения оверлэпа для вставок в средней 
точке и вторую (обозначенную как $(2))$ - без него ${ }^{1)}$. Обе они отличаются от схемы, предложенной в работе [13], тем, как мы обращается с нулевыми духовыми модами. Эти схемы дают соотношение спуска в виде

$$
\left\langle V_{3}^{\mathrm{m}} \mid V_{1}^{\mathrm{m}}\right\rangle=\widetilde{Z}_{3}^{\mathrm{m}}(n)\left\langle V_{2}^{\mathrm{m}}\right|, \quad\left\langle V_{3}^{\mathrm{gh}} \mid V_{1}^{\mathrm{gh}}\right\rangle_{(i)}=\widetilde{Z}_{3(i)}^{\mathrm{gh}}(n)\left\langle V_{2}^{\mathrm{gh}}\right|, \quad i=1,2,
$$

где $\widetilde{Z}_{3}^{\mathrm{m}}(n)$ и $\widetilde{Z}_{3}^{\mathrm{gh}}(n)$ - константы, зависящие от уровня обрезания $n$. Для $n \rightarrow \infty$ материальная часть $\widetilde{Z}_{3}^{\mathrm{m}}(n) \rightarrow 0$, а духовая $\widetilde{Z}_{3}^{\text {gh }}(n) \rightarrow \infty$ для обеих схем счета. Собирая материальную и духовую части, мы получаем нормировку $\widetilde{Z}_{3}$ как $\widetilde{Z}_{3}(n)=$ $\widetilde{Z}_{3}^{\text {gh }}(n) \widetilde{Z}_{3}^{\mathrm{m}}(n)$. Численно мы докажем, что $\widetilde{Z}_{3}(n)$ имеет конечный предел при $n \rightarrow \infty$ для обеих схем. Удивительным фактом является то, что для двух различных схем мы получаем два различных ответа, т.е. $\widetilde{Z}_{3(1)}(\infty) \cong 0.070075$ и $\widetilde{Z}_{3(2)}(\infty) \cong 0.199381$. Значение $\widetilde{Z}_{3(2)}(\infty)$ согласуется с результатом, полученным в работе [13].

\section{2. ПОСТАНОВКА ЗАДАЧИ}

В операторном представлении струнные поля $\Phi$ реализуются как кет-векторы $|\Phi\rangle$ в фоковском пространстве $\mathcal{H}$ материальных и духовых мод. В дальнейшем обсуждении удобно использовать графическое представление, аналогичное стандартной диаграммной технике, которая используется для вычисления вакуумного среднего произведения мономов Вика. Мономы Вика представляются стрелкой. Таким образом, операторы рождения соответствуют стрелкам, направленным налево, а операторам уничтожения - стрелкам, направленным направо. Здесь для простоты мы будем использовать одну стрелку для всех операторов рождения или уничтожения. Таким образом, мы представляем бра-векторы точкой со стрелкой, направленной направо и кет-векторы - точкой со стрелкой, направленной налево, т.е. состояние $|\Phi\rangle$ представляется как

$$
|\Phi\rangle=\longleftarrow \cdot
$$

Для того чтобы построить действие Виттена (1.1) [1], необходимо и достаточно ввести только две вершины:

$$
{ }_{12}\left\langle\left|V_{3}\right|\right\rangle_{3} \in \mathcal{H}^{*} \otimes \mathcal{H}^{*} \otimes \mathcal{H}
$$

представляющую $\star$, и

$$
\left\langle V_{1}\right| \in \mathcal{H}^{*}
$$

которая представляет $\int$.

В графических обозначениях $\left\langle V_{1}\right|$ представляется кружочком со стрелкой напраBO:

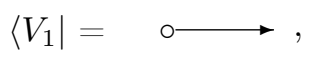

1) Мы специально изучаем два способа вычисления, поскольку это позволяет прояснить вопрос о возможности переноса вставки с учетом оверлэпа. Этот вопрос особенно важен в связи с тем, что использование вставок неизбежно для случая суперструн [17]. 
и $\left\langle V_{1} \mid \Phi\right\rangle-$ как

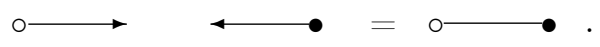

Виттеновская вершина ${ }_{12}\left\langle\left|V_{3}\right|\right\rangle_{3}$ представляется тремя стрелками, имеющими общее начало. Две из них идут направо, а третья - налево. Таким образом, мы можем представить произведение "звездочка" в виде

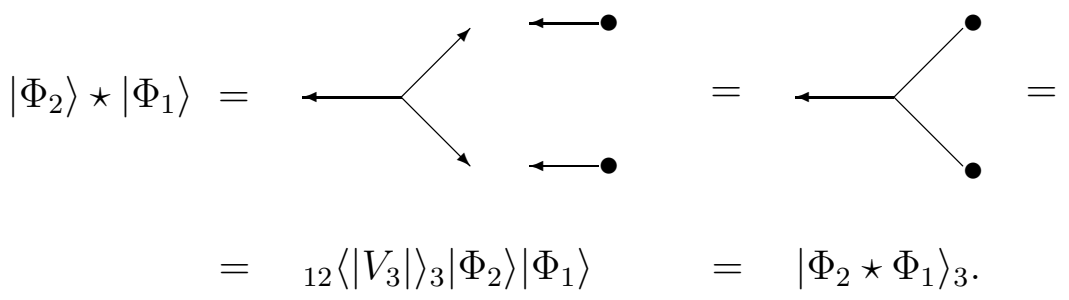

Можно построить бесконечную башню вершин ${ }_{1 \ldots N}\left\langle\left|V_{N+1}\right|\right\rangle_{N+1}$ склейкой $N-1$ вершин $V_{3}$. Графически эта башня имеет вид дерева с $N+1$ стрелками. Внутри этого дерева мы можем склеивать свободные концы вершин $V_{3}$ в произвольном порядке. Все эти склейки эквивалентны (это является следствием ассоциативности произведения "звездочка" [2]), и в дальнейшем мы будем иметь в виду самый простой вариант:

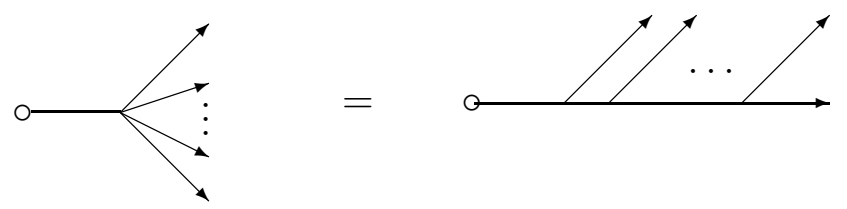

Используя вершину $1 \ldots N\left\langle\left|V_{N+1}\right|\right\rangle_{N+1}$, можно определить звездочное произведение $N$ полей как

$$
{ }_{1 \ldots N}\left\langle\left|V_{N+1}\right|\right\rangle_{N+1}\left|\Phi_{N}\right\rangle \ldots\left|\Phi_{1}\right\rangle \equiv\left|\Phi_{N}\right\rangle \star \cdots \star\left|\Phi_{1}\right\rangle .
$$

Левая часть уравнения (2.1) может быть представлена в виде

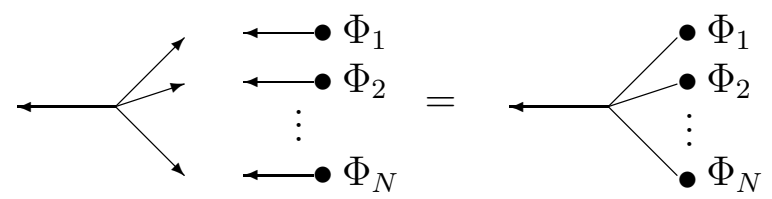

Можно также построить бесконечную башню вершин ${ }_{1 \ldots N}\left\langle V_{N}\right|$, которые получаются из сконструированных выше вершин ${ }_{1 \ldots N}\left\langle\left|V_{N+1}\right|\right\rangle_{N+1}$ добавлением вершины $\left\langle V_{1}\right|$, как показано ниже:

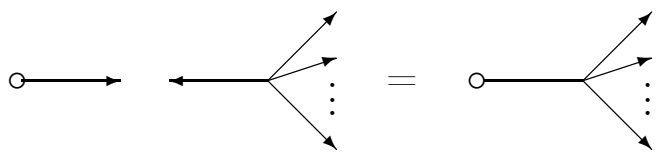


Назовем набор таких вершин виттеновской башней вершин и обозначим их шляпкой. Для согласованности также шляпкой обозначим первоначальные вершины $\left\langle V_{1}\right|$ и $\left\langle\left|V_{3}\right|\right\rangle$. Виттеновская башня определяется вершинами $\left\langle\widehat{V}_{1}\right|$ и $\left\langle\left|\widehat{V}_{3}\right|\right\rangle$, которые определены с точностью до числа. Поэтому нам нужно только два условия, чтобы однозначно фиксировать виттеновскую башню. Наиболее естественный путь - постулировать соответствие струнно-полевых вершин и корреляторов в конформной теории поля:

$$
\left\langle\widehat{V}_{N} \mid \Phi_{1}\right\rangle \ldots\left|\Phi_{N}\right\rangle \equiv\left\langle\Phi_{1} \ldots \Phi_{N}\right\rangle_{\Sigma_{N}}, \quad N=2,3,
$$

где $\Sigma_{N}$ - соответствующая риманова поверхность [3]. Не ясно, гарантирует ли данная фиксация неопределенности выполнение условия (2.2) для всех вершин $(N>3)$ в башне.

Мы также находим кет-вершину $\left|\widehat{V}_{1}\right\rangle$ как решение соотношения спуска [2]

$$
\left\langle\widehat{V}_{2} \mid \widehat{V}_{1}\right\rangle=\left\langle\widehat{V}_{1}\right| \text {. }
$$

Такое определяющее уравнение для $\left|\widehat{V}_{1}\right\rangle$ может быть представлено графически. При этом мы представляем вершину, соответствующую $\left|\widehat{V}_{1}\right\rangle$, звездочкой $*$ со стрелкой налево. Теорема Вика позволяет левую часть уравнения (2.3) представить в виде

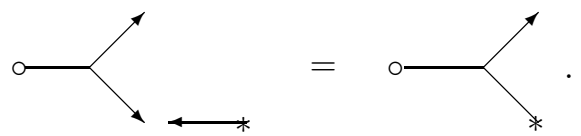

В этих обозначениях уравнение (2.3) выглядит как

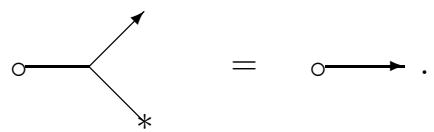

С помощью $\left|\widehat{V}_{1}\right\rangle$, определенной уравнением (2.3), мы будем проверять соотношения спуска

$$
\left\langle\widehat{V}_{N+1} \mid \widehat{V}_{1}\right\rangle=\left\langle\widehat{V}_{N}\right|
$$

для $N>1$, которое может быть графически представлено в виде
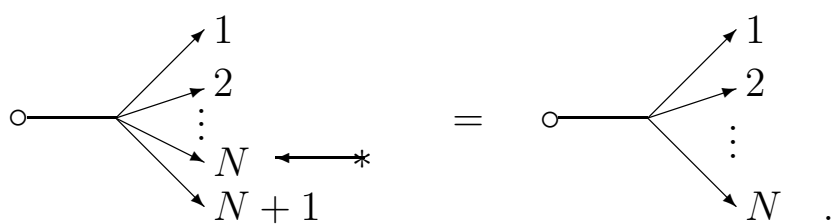

Для того чтобы доказать соотношение (2.4), мы должны использовать нашу конструкцию вершин $\left\langle\widehat{V}_{N+1}\right|$ (как отмечено выше, мы используем самый простой граф), т.e.

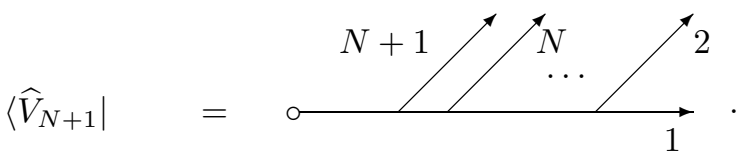


Далее,

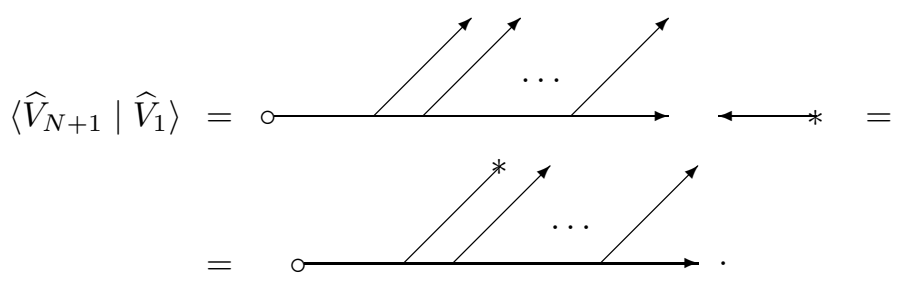

Принимая во внимание уравнение (2.3), получаем,
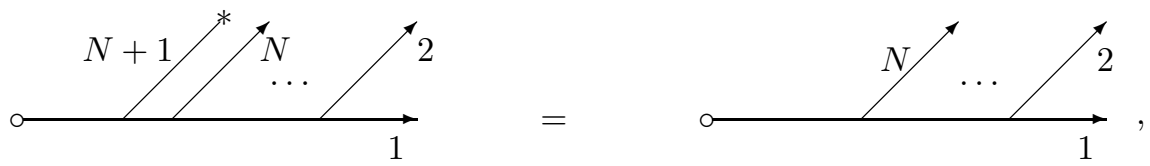

что в точности дает представление для $\left\langle\widehat{V}_{N}\right|$.

Бра-вершины $\left\langle V_{i}\right|$ обычно ищутся как решения условий оверлэпа [2], [6], [8], [18]. Однако из-за однородности условий оверлэпа эти решения определены с точностью до численного фактора, т.е. вершина $\left\langle\widehat{V}_{N}\right|$ в виттеновской башне отличается от любой другой вершины $\left\langle V_{N}\right|:\left\langle\widehat{V}_{N}\right|=Z_{N}\left\langle V_{N}\right|$ и $\left|\widehat{V}_{1}\right\rangle=Z_{-1}\left|V_{1}\right\rangle$. Таким образом, соотношения спуска для $\left\langle V_{N}\right|$ становятся соотношениями (1.3).

Факторизация коэффициента в соотношении спуска была впервые найдена в работе [15], где вершины строились в непрерывном $\kappa$-базисе. Оказалось, что соотношение спуска содержит коэффициент, не равный единице, и, более того, этот коэффициент допускает факторизацию на три сомножителя. Каждый из этих сомножителей интерпретировался как граничная энтропия конформной теории поля. С использованием этой факторизации вершины были переопределены как $\left\langle\left\langle V_{N}\right|=Z_{N}\left\langle V_{N}\right|\right.$. После такого переопределения соотношение спуска имеет коэффициент, равный единице: $\left\langle\left\langle V_{N+1} \mid V_{1}\right\rangle\right\rangle=\left\langle\left\langle V_{N}\right|\right.$.

\section{3. СООТНОШЕНИЯ СПУСКА ДЛЯ $N=1$ И $N=2$}

3.1. $\left\langle V_{2} \mid V_{1}\right\rangle$. Вершины, которые будут использоваться ниже, были построены в работах [2], [5]-[7], [18]. Здесь и ниже мы используем векторные обозначения для мод полей материи $X^{\mu}(\sigma)$ как $^{2)} a=\left(a_{0}, a_{1}, \ldots\right), a^{*}=\left(a_{0}^{*}, a_{-1}, \ldots\right)$ и для духовых полей $b(\sigma), c(\sigma)$ как $b, b^{*}$ и $c, c^{*}$, соответственно (см. приложение). В некоторых отдельно оговоренных случаях мы будем включать нулевую моду $b_{0}$ в $b$. Нулевая мода $c_{0}$ не входит ни в $c$, ни в $c^{*}$. Также мы используем матрицу $S_{n k}=(-)^{n+1} \delta_{n k}$, где $n, k \geqslant 0$.

Начнем вычисления с материального сектора. Точное осцилляторное представление для $V_{1}$ и $V_{2}$ имеет вид

$$
\left|V_{1}^{\mathrm{m}}\right\rangle=e^{a^{* 1} S a^{* 1} / 2}|0\rangle_{1}, \quad\left\langle V_{2}^{\mathrm{m}}\right|={ }_{1,2}\langle 0| e^{a^{1} S a^{2}}, \quad\left\langle V_{1}^{\mathrm{m}}\right|={ }_{1}\langle 0| e^{a^{1} S a^{1} / 2} .
$$

Левая часть уравнения (1.3) есть

$$
\left\langle V_{2}^{\mathrm{m}} \mid V_{1}^{\mathrm{m}}\right\rangle={ }_{1,2}\left\langle 0\left|e^{a^{1} S a^{2}} e^{a^{1 *} S a^{1 *} / 2}\right| 0\right\rangle_{1}={ }_{2}\langle 0| e^{a^{2} S S S a^{2} / 2}={ }_{2}\langle 0| e^{a^{2} S a^{2} / 2}=\left\langle V_{1}^{\mathrm{m}}\right| .
$$

\footnotetext{
2) Далее мы опускаем пространственно-временной индекс.
} 
Здесь мы использовали теорему Вика [19].

Теперь рассмотрим соотношение спуска в духовом секторе. Для этих вычислений используем следующие вершины (в этом случае $n, m \geqslant 1$ ):

$$
\begin{gathered}
\left|V_{1}^{\mathrm{gh}}\right\rangle=\frac{i}{4} b_{+}^{1} b_{-}^{1} e^{b^{* 1} S c^{* 1}}|+\rangle_{1}, \quad\left\langle V_{1}^{\mathrm{gh}}\right|={ }_{1}\langle+| e^{-b^{1} S c^{1}} b_{-}^{1} b_{+}^{1} \frac{i}{4}, \\
\left\langle V_{2}^{\mathrm{gh}}\right|={ }_{1,2}\langle+|\left(b_{0}^{1}-b_{0}^{2}\right) e^{-b^{1} S c^{2}-b^{2} S c^{1}} .
\end{gathered}
$$

Вставки в средней точке суть

$$
b_{+} \equiv b\left(\frac{\pi}{2}\right)=\sum_{-\infty}^{\infty} i^{n} b_{n}, \quad b_{-} \equiv b\left(-\frac{\pi}{2}\right)=\sum_{-\infty}^{\infty} i^{-n} b_{n} .
$$

Перепишем левую часть соотношения спуска в виде

$$
\left\langle V_{2}^{\mathrm{gh}} \mid V_{1}^{\mathrm{gh}}\right\rangle={ }_{1,2}\left\langle+\left|\left(b_{0}^{1}-b_{0}^{2}\right) e^{-b^{1} S c^{2}-b^{2} S c^{1}} \frac{i}{4} b_{+}^{1} b_{-}^{1} e^{b^{1 *} S c^{1 *}}\right|+\right\rangle_{1} .
$$

Будем использовать условие оверлэпа для $b_{+}$и $b_{-}$, чтобы изменить индекс вставки c "1" на "2". Для того чтобы изменить порядок вакуумных состояний, мы должны принять во внимание их четность, т.е.

$$
{ }_{1,2}\langle+|=-{ }_{2,1}\langle+| \text {. }
$$

Таким образом, используя полученные в работе [19] формулы, имеем

$$
\left\langle V_{2}^{\mathrm{gh}} \mid V_{1}^{\mathrm{gh}}\right\rangle={ }_{-2}\langle+| e^{-b^{2} S S S c^{2}} b_{+}^{2} b_{-}^{2} \frac{i}{4}={ }_{-2}\langle+| e^{-b^{2} S c^{2}} b_{+}^{2} b_{-}^{2} \frac{i}{4}=\left\langle V_{1}^{\mathrm{gh}}\right| .
$$

Итак, для вершин, включающих материальные и духовые секторы, соотношение спуска с коэффициентом $\widetilde{Z}_{2}$, равным единице, имеет вид

$$
\left\langle V_{2} \mid V_{1}\right\rangle=\left\langle V_{1}\right| \text {. }
$$

Мы можем заключить, что вершины $\left\langle V_{1}\right|,\left\langle V_{2}\right|$ и $\left|V_{1}\right\rangle$ могут рассматриваться как вершины из некоторой виттеновской башни.

3.2. $\left\langle V_{3} \mid V_{1}\right\rangle$. В этом пункте мы рассмотрим соотношение спуска для вершин $\left\langle V_{3}\right|$ и $\left|V_{1}\right\rangle$. Это вычисление не такое простое, как представленное выше.

Проведем вычисления соотношения спуска, начиная с духового сектора (обозначения см. в приложении):

$$
\left\langle V_{3}^{\mathrm{gh}} \mid V_{1}^{\mathrm{gh}}\right\rangle=N_{3} \frac{i}{4} 321\left\langle+\left|e^{-b^{r} X^{r s} c^{s}} b_{+}^{1} b_{-}^{1} e^{b^{* 1} S c^{* 1}}\right|+\right\rangle_{1} .
$$

Здесь мы включаем нулевую моду $b_{0}$ в $b$. Опустим в вычислениях коэффициент $(i / 4) N_{3}=(i / 4)(3 \sqrt{3}) / 4$. Он будет восстановлен в конечном выражении.

Рассмотрим две схемы вычисления соотношения (3.3). Можно оставить вставку $b_{+}^{1} b_{-}^{1}$ в том виде, как она есть, либо использовать одно из условий оверлэпа для вершины $\left\langle V_{3}^{\mathrm{gh}}\right|$

$$
\left\langle V_{3}^{\mathrm{gh}}\right|\left(b^{i-1}(\sigma)-b^{i}(\pi-\sigma)\right)=0, \quad i=1,2,3,
$$


чтобы переместить $b_{+}^{1}$ и $b_{-}^{1}$ в фоковское пространство с индексом " 2 " или "3". Первая схема вычисления будет представлена в п. 3.2.2, а вторая - в п 3.2.1.

В работе [13] была представлена другая схема: вершина $\left|V_{1}\right\rangle$ строилась над $|-\rangle$ вакуумом, и при проверке соотношения (1.4) были проведены прямые вычисления.

3.2.1. “Оверлэп вычисления” в секторе духов. Мы используем условие оверлэпа, чтобы изменить индекс вставки $b_{+}$с "1" на "2" и $b_{-}-$с "1" на "3". Таким образом, мы должны вычислить выражение (здесь $b^{r}$ включает $b_{0}^{r}$ )

$$
{ }_{321}\left\langle+\left|e^{-b^{r} X^{r s} c^{s}} e^{b^{* 1} S c^{* 1}}\right|+\right\rangle_{1} b_{+}^{2} b_{-}^{3} .
$$

Отделяя нулевую моду $b_{0}^{1}$, получаем

$$
\begin{gathered}
{ }_{32}\langle+| e^{-W_{2}}{ }_{1}\left\langle+\left|\left(1-b_{0} w\right) e^{-W_{1}} e^{b^{* 1} S c^{* 1}}\right|+\right\rangle_{1} b_{+}^{2} b_{-}^{3}= \\
=-{ }_{32}\langle+| e^{-W_{2}}{ }_{1}\left\langle-\left|e^{-W_{1}} w e^{b^{* 1} S c^{* 1}}\right|+\right\rangle_{1} b_{+}^{2} b_{-}^{3},
\end{gathered}
$$

где мы положили

$$
w=X_{0 m}^{11} c_{m}^{1}+X_{0 m}^{1 p} c_{m}^{p}, \quad p=2,3,
$$

в $W_{1}$ собрали зависимость от ненулевых мод $b^{1}, c^{1}$, а в $W_{2}$ - от мод второй и третьей струн (см. приложение).

Зависимость от нулевой моды здесь - предмет особого внимания. В наших вычислениях для любой матрицы $A_{n m}$ мы обозначаем $\bar{A}_{k} \equiv A_{0 k}$ и $\hat{A}_{n m} \equiv A_{n m}$, где $n>0$.

Таким образом, необходимо вычислить следующее выражение:

$$
{ }_{1}\left\langle-\left|e^{-W_{1}} w e^{b^{* 1}} S c^{* 1}\right|+\right\rangle_{1} .
$$

Для этого мы используем обобщенную формулу (19) из работы [19], которая была приведена в работах [13], [20],:

$$
\begin{aligned}
& \left\langle-\left|e^{-b X c+b \lambda^{c}+\lambda^{b} c} e^{b^{*} S c^{*}+b^{*} \mu^{c}+\mu^{b} c^{*}}\right|+\right\rangle= \\
& \quad=\operatorname{det}(1-S X) \exp \left\{\mu^{b} \frac{1}{1-X S}\left(X \mu^{c}-\lambda^{c}\right)+\lambda^{b} \frac{1}{1-S X}\left(\mu^{c}-S \lambda^{c}\right)\right\} .
\end{aligned}
$$

Здесь $\lambda^{b}, \lambda^{c}$ и $\mu^{b}, \mu^{c}-$ антикоммутирующие векторы.

Таким образом, используя (3.7) и $3,2\langle+|=-{ }_{2,3}\langle+|$, мы можем вычислить $(3.6)$, так что выражение (3.5) принимает вид

$$
\left\langle V_{3}^{\mathrm{gh}} \mid V_{1}^{\mathrm{gh}}\right\rangle=-\operatorname{det}\left(Z^{-1}\right)_{23}\langle+| \bar{U}^{1 q} c^{q} e^{b^{p} U^{p q} c^{q}} b_{+}^{2} b_{-}^{3},
$$

где

$$
U_{n m}^{p q}=-X_{n k}^{p 1}(Z S)_{k l} X_{l m}^{1 q}-X_{n m}^{p q},
$$

$n \geqslant 0, m, k, l \geqslant 1, p, q=2,3$.

Здесь мы определили

$$
Z=(1-S X)^{-1},
$$


где $X_{n m} \equiv X_{n m}^{11}$, и хотим отметить, что $n, m \geqslant 1$.

Чтобы привести выражение (3.8) к нормальной форме, перемещаем вставку $b_{+}^{2} b_{-}^{3}$ налево к вакууму через экспоненту и предэкспоненту. В результате получаем следующий предэкспоненциальный фактор (обозначения см. в приложении):

$$
\begin{aligned}
\left(b^{2} K\right. & \left.+b_{0}^{2}+b_{0}^{p} \bar{U}^{p 2} L+b^{p} \widehat{U}^{p 2} L\right)\left(b^{3} L+b_{0}^{3}+b_{0}^{p} \bar{U}^{p 3} K+b^{p} \widehat{U}^{p 3} K\right) \bar{U}^{1 q} c^{q}- \\
& -\left(b^{2} K+b_{0}^{2}+b_{0}^{p} \bar{U}^{p 2} L+b^{p} \widehat{U}^{p 2} L\right) \bar{U}^{13} K+\left(b^{3} L+b_{0}^{3}+b_{0}^{p} \bar{U}^{p 3} K+b^{p} \widehat{U}^{p 3} K\right) \bar{U}^{12} L .
\end{aligned}
$$

Так как вакуумы находятся слева, слагаемые с операторами рождения удалены.

Матрицы $\widehat{U}^{p q}$ и строки $\bar{U}^{p q}$ для различных значений $p$ и $q$ не независимы. Согласно определению (3.9) и свойствам матриц Неймана (см. приложение), имеем

$$
\begin{aligned}
& \widehat{U}^{i 2}+\widehat{U}^{i 3}=-S, \quad \widehat{U}^{2 i}+\widehat{U}^{3 i}=-S, \quad i=2,3, \\
& \bar{U}^{1 j}+\bar{U}^{2 j}+\bar{U}^{3 j}=0, \quad \bar{U}^{j 2}+\bar{U}^{j 3}=0, \quad j=1,2,3 \text {. }
\end{aligned}
$$

Для примера можно доказать первое свойство [13]:

$$
\begin{aligned}
\widehat{U}^{i 2}+\widehat{U}^{i 3} & =-X^{i 2}-X^{i 1}\left(1-S X^{11}\right)^{-1} S X^{12}-X^{i 3}-X^{i 1}\left(1-S X^{11}\right)^{-1} S X^{13}= \\
& =-X^{i 2}-X^{i 3}-X^{i 1}\left(1-S X^{11}\right)^{-1} S\left(X^{12}+X^{13}\right)= \\
& =-X^{i 2}-X^{i 3}-X^{i 1}\left(1-S X^{11}\right)^{-1} S\left(S-X^{11}\right)= \\
& =-X^{i 2}-X^{i 3}-X^{i 1}\left(1-S X^{11}\right)^{-1}\left(1-S X^{11}\right)=-S .
\end{aligned}
$$

Используя соотношения (3.11), выражение (3.10) можно упростить. Мы выражаем все матрицы через $\widehat{U}^{22}$.

Удобно ввести следующие обозначения:

$$
\begin{array}{ll}
\alpha_{2}=\left(b^{2}+b^{3}\right) K+\left(b^{2}-b^{3}\right) \widehat{U}^{22} L, & \beta_{2}=b_{0}^{2}\left(1+\bar{U}^{22} L\right)+b_{0}^{3} \bar{U}^{32} L, \\
\alpha_{3}=\left(b^{2}+b^{3}\right) L-\left(b^{2}-b^{3}\right) \widehat{U}^{22} K, & \beta_{3}=b_{0}^{3}\left(1+\bar{U}^{33} K\right)+b_{0}^{2} \bar{U}^{23} K .
\end{array}
$$

Таким образом, результат переноса вставки $b_{+} b_{-}$налево (предэкспоненциальный фактор (3.10)) можно представить как

$$
\left(\alpha_{2}+\beta_{2}\right)\left(\alpha_{3}+\beta_{3}\right) \bar{U}^{1 q} c^{q}-\left(\alpha_{2}+\beta_{2}\right) \bar{U}^{13} K+\left(\alpha_{3}+\beta_{3}\right) \bar{U}^{12} L .
$$

Результат взятия среднего и приведения выражения к нормальной форме имеет вид

$$
-\operatorname{det}\left(Z^{-1}\right)_{23}\langle+|\left[\left(\alpha_{2}+\beta_{2}\right)\left(\alpha_{3}+\beta_{3}\right) \bar{U}^{1 q} c^{q}-\left(\alpha_{2}+\beta_{2}\right) \bar{U}^{13} K+\left(\alpha_{3}+\beta_{3}\right) \bar{U}^{12} L\right] e^{b^{p} U^{p q} c^{q} .}
$$

На первый взгляд, этот результат не похож на вершину $\left\langle V_{2}^{\text {gh }}\right|$, так как не имеет правильной структуры вакуума и правильного показателя экспоненты. Ниже мы покажем, как можно восстановить из него правильный вид вершины $\left\langle V_{2}^{\mathrm{gh}}\right|$.

При вычислениях будут полезны следующие тождества, установленные численно для любого уровня:

$$
\bar{U}^{23}=-\bar{U}^{32} S, \quad \bar{U}^{22}=-\bar{U}^{33} S, \quad \bar{U}^{12}=-\bar{U}^{13} S .
$$


Отсюда можно получить

$$
\bar{U}^{32} L=\bar{U}^{23} K, \quad \bar{U}^{22} L=\bar{U}^{33} K, \quad \bar{U}^{13} K=\bar{U}^{12} L, \quad \bar{U}^{32} L=-\bar{U}^{22} K .
$$

В выражении (3.14) нам встретится столбец $\widehat{U}^{22}(L+K)$, который мы рассмотрим численно. Подставляя точный вид $L$ и $K$, получим элемент столбца в виде

$$
a_{m}=2 \sum_{k=0}^{\infty}(-)^{k} \widehat{U}_{m, 2 k}^{22} .
$$

Для произвольного уровня $n$ вычислим частную сумму

$$
a_{m}(n)=2 \sum_{k=0}^{n}(-)^{k} \widehat{U}_{m, 2 k}^{22}
$$

при $m \leqslant n$. Для любого фиксированного $m$ получаем последовательность частных сумм $a_{m}(n)$ как функцию $n$. Докажем, что $a_{m}(n) \rightarrow 0$ при $n \rightarrow \infty$. Покажем это для элементов столбца с номерами $m=2,10,20,30, \ldots, 100$ для уровней $100 \leqslant n \leqslant 300$ с шагом 10. Результат представлен на рис. 1. Найдем также функцию, описывающую эти графики, в виде $\left|a_{m}(n)\right| \sim p_{m} / n^{q_{m}}$ (см. таблицу). Из вида этих функций делаем вывод, что $\left|a_{m}(n)\right| \rightarrow 0$ при $n \rightarrow \infty$ и, как следствие, $a_{m} \rightarrow 0$. Из результатов, приведенных в таблице и на рис. 1 , следует, что скорость убывания элементов $a_{m}$ растет с увеличением номера $m$. Следовательно,

$$
\widehat{U}^{22}(L+K)=0 .
$$

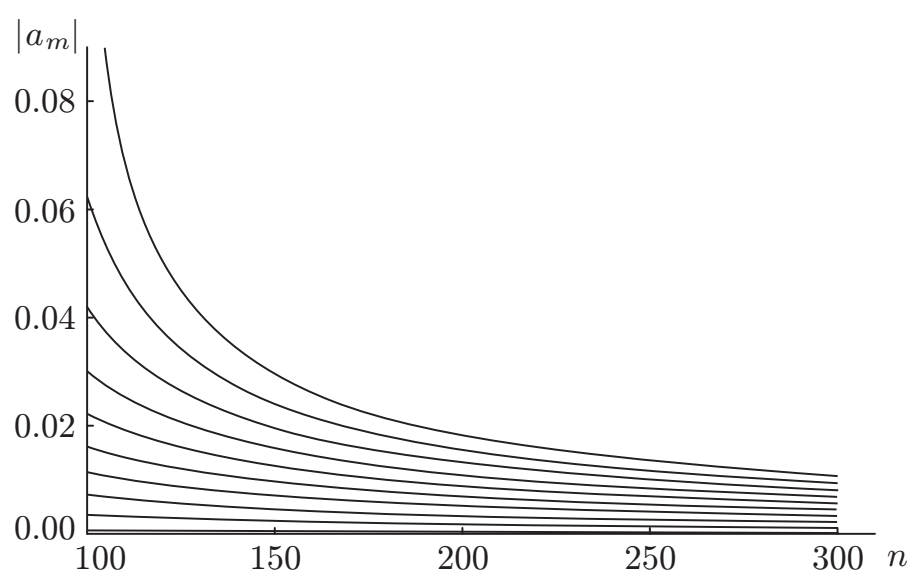

Рис. 1

Вернемся к выражению (3.14). Подставим в него выражения (3.13) и выделим слагаемые, линейные по $b_{0}$. Учитывая свойства (3.17) и (3.18), получим

$$
\left(b_{0}^{2}\left[J_{0}+\left(b^{2} \bar{A}_{2}+b^{3} \bar{A}_{3}\right) \bar{U}^{1 q} c^{q}\right]-b_{0}^{3}\left[J_{0}+\left(b^{2} \bar{B}_{2}+b^{3} \bar{B}_{3}\right) \bar{U}^{1 q} c^{q}\right]\right),
$$




\begin{tabular}{|c|c|c|c|c|c|c|}
\hline$m$ & 2 & 10 & 20 & 30 & 40 & 50 \\
\hline$p$ & 0.089 & 0.4617 & 1.0258 & 1.844 & 3.2117 & 5.806 \\
\hline$q$ & 1.053 & 1.059 & 1.077 & 1.109 & 1.156 & 1.221 \\
\hline
\end{tabular}

\begin{tabular}{|c|c|c|c|c|c|}
\hline$m$ & 60 & 70 & 80 & 90 & 100 \\
\hline$p$ & 11.463 & 26.227 & 76.781 & 364.31 & 16461 \\
\hline$q$ & 1.309 & 1.428 & 1.595 & 1.854 & 2.529 \\
\hline
\end{tabular}

где (мы выразили все строки через строки $\bar{U}^{12}$ и $\bar{U}^{22}$, используя свойства (3.17))

$$
\begin{aligned}
& J_{0}=-\left(1+\bar{U}^{22}(K+L)\right) \bar{U}^{12} L, \\
& \bar{A}^{i}=(-)^{i} \widehat{U}^{22} L\left(1+\bar{U}^{22}(K+L)\right)+L\left(1+\bar{U}^{22} L\right)+K \bar{U}^{22} K, \\
& \bar{B}^{i}=(-)^{i} \widehat{U}^{22} L\left(1+\bar{U}^{22}(K+L)\right)+K\left(1+\bar{U}^{22} L\right)+L \bar{U}^{22} K .
\end{aligned}
$$

Таким образом, величина $J_{0}$ дает вклад в нормировочный множитель $\widetilde{Z}_{3}^{\text {gh }}(n)$. Оставшиеся слагаемые, квадратичные по $b_{0}$, запишем в виде

$$
\beta_{2} \beta_{3} \bar{U}^{1 q} c^{q}=b_{0}^{2} b_{0}^{3}\left[\left(1+\bar{U}^{22} L\right)^{2}-\left(\bar{U}^{22} K\right)^{2}\right] \bar{U}^{1 q} c^{q} .
$$

В выражение (3.14) входят два слагаемых, не содержащих $b_{0}$ и стремящихся к нулю в масштабе $J_{0}$. На рис. 2 показано поведение этих слагаемых (рис. $2 \mathrm{a}-\alpha_{2} \alpha_{3} \bar{U}^{1 q} c^{q}$, рис. $\left.2 б-\left(\alpha_{3}-\alpha_{2}\right) \bar{U}^{12} L\right)$ нормированных на $J_{0}$ при $n \rightarrow \infty$. Численно установлено, что на уровнях $n=20, \ldots, 300$ они описываются формулами $\sim 0.87 / n^{0.99}$ и $\sim 0.41 / n^{0.9}$, соответственно. Из графиков видно, что эти кривые стремятся к нулю при $n \rightarrow \infty$.

Теперь мы можем поднять слагаемые, линейные и квадратичные по $b_{0}$, из предэкспоненты в показатель экспоненты и, используя эту добавку, получить правильную структуры экспоненты:

$$
{ }_{23}\langle+| J_{0}(\ldots) e^{b U c} \Longrightarrow{ }_{23}\langle+| J_{0}\left(b_{0}^{2}-b_{0}^{3}\right) e^{b V c} e^{b U c}
$$

(здесь нулевые моды входят как в матрицу $V$, так и в матрицу $U$ ). Для получения правильного показателя экспоненты необходимо выполнение следующих условий (при $n \rightarrow \infty)$ :

$$
\begin{gathered}
\bar{V}^{p q}+\bar{U}^{p q} \rightarrow 0, \\
\widehat{V}^{p p}+\widehat{U}^{p p} \rightarrow 0, \quad \widehat{V}^{p, p+1}+\widehat{U}^{p, p+1} \rightarrow-S .
\end{gathered}
$$

Условие (3.23) убирает все нулевые моды в показателе экспоненты, а условие (3.24) обеспечивает правильную матричную структуру. Ниже мы покажем, как это сделать.

Удобно провести обратное действие, т.е. разложить экспоненту $e^{b V c}$ и сравнить получившуюся структуру с полученной ранее в выражении (3.14). Таким образом, 

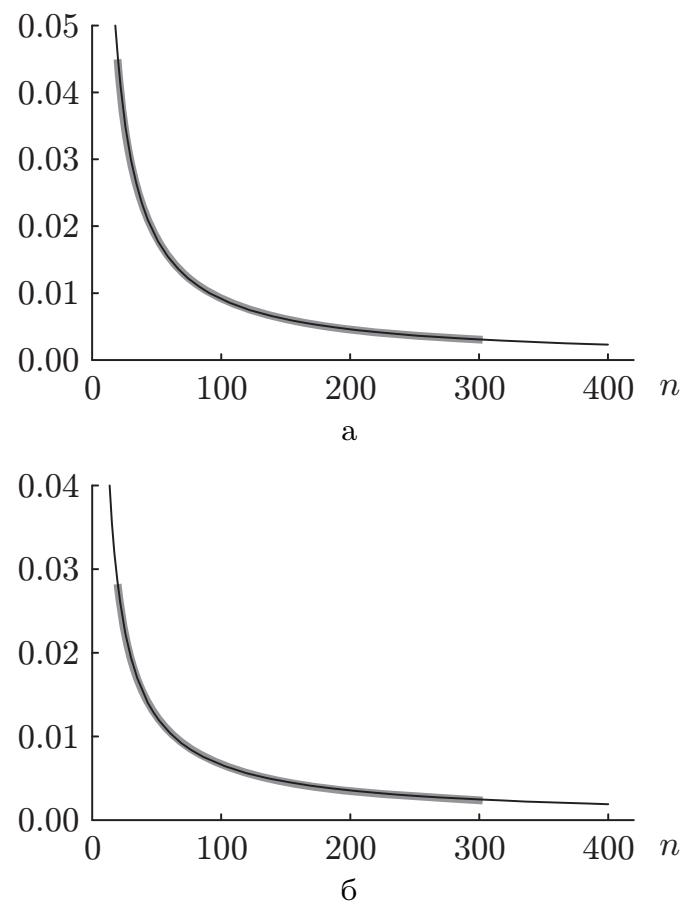

Рис. 2

получим условия для предэкспоненты, необходимые для ее поднятия в показатель экспоненты. Здесь важно предположить, что матрица $\widehat{V}$ представима как прямое произведение двух векторов, стоящих при нулевых модах, т.е. $\widehat{V}=\bar{V}_{1} \otimes \bar{V}_{2}$. Из этого предположения следует, что экспонента $e^{b V c}$ разлагается только до линейных членов (остальные слагаемые равны нулю по грассмановости).

Таким образом, имеется следующая система для неизвестных матриц $V^{p q}, p, q=$ 2,3 :

$$
\begin{gathered}
J_{0}\left(\bar{V}^{2 q}+\bar{V}^{3 q}\right)=\left[\left(1+\bar{U}^{22} L\right)^{2}-\left(\bar{U}^{22} K\right)^{2}\right] \bar{U}^{1 q} \\
\left(\bar{V}^{3 q}+\bar{V}^{2 q}\right) c^{q} \cdot b^{p} \widehat{V}^{p q} c^{q}=0 \\
\widehat{V}^{p q}=\frac{\bar{A}^{p}}{J_{0}} \otimes \bar{U}^{1 q} \\
\widehat{V}^{p q}=\frac{\bar{B}^{p}}{J_{0}} \otimes \bar{U}^{1 q}
\end{gathered}
$$

Эти условия не определяют матрицу $V^{p q}$ полностью, но их достаточно, чтобы поднять предэкспоненту в показатель экспоненты.

Условие совместности этой системы имеет вид

$$
\frac{\bar{A}^{p}}{J_{0}}-\frac{\bar{B}^{p}}{J_{0}}=T \frac{\left(1+\bar{U}^{22} T\right)}{J_{0}} \rightarrow 0, \quad p=2,3 .
$$


Численное вычисление, проведенное для этой величины на уровнях $n=20, \ldots, 300$, показывает, что она описывается функцией $4.3 / n^{0.94}$ (см. рис. 3 ).

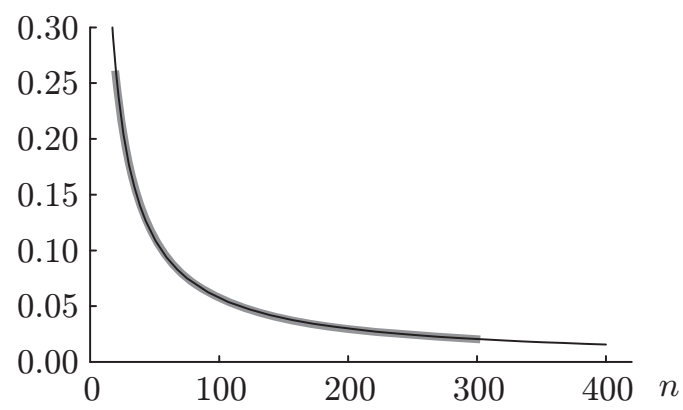

Рис. 3

Уравнение (3.25в) определяет матрицу $\widehat{V}^{p q}$, а условие $(3.25 \mathrm{a})-$ сумму $\bar{V}^{2 q}+\bar{V}^{3 q}$. Условие (3.25б) следует из (3.25a) и (3.25в):

$$
\frac{1}{J}_{0}\left[\left(1+\bar{U}^{22} L\right)^{2}-\left(\bar{U}^{22} K\right)^{2}\right] \bar{U}^{1 q} c^{q} b^{2} \frac{\bar{A}^{p}}{J_{0}} \otimes \bar{U}^{1 q} c^{q}=0 .
$$

Условие (3.25а) определяет не матрицы $\bar{V}^{p q}$, а только их сумму. Положив $\bar{V}^{p q} \equiv$ $-\bar{U}^{p q}$, мы удовлетворим условию (3.23). Сделав такой выбор, рассмотрим условие (3.25a):

$$
-J_{0}\left(\bar{U}^{2 q}+\bar{U}^{3 q}\right)-\left[\left(1+\bar{U}^{22} L\right)^{2}-\left(\bar{U}^{22} K\right)^{2}\right] \bar{U}^{1 q}=0
$$

Учитывая соотношения (3.12) и сократив столбец $\bar{U}^{1 q}$ в равенстве $(3.27)$, проверим численно его выполнение. На рис. 4 показано поведение величины (3.27) в зависимости от уровня (до $n=300$ ); кривая описывается функцией $3.16 / n^{0.89}$.

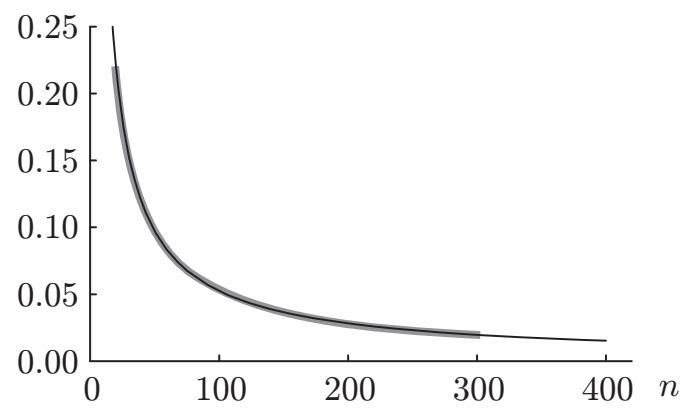

Рис. 4

Условие (3.25в) определяет матрицу $\widehat{V}^{p q}=J_{0}^{-1} \bar{A}^{p} \otimes \bar{U}^{1 q}$. Проверим выполнение условий (3.24). При $p=2$ для первого из них мы получаем функцию $\sim 0.86 / n^{0.82}$ (для второго из условий (3.24) и для $p=3$ вычисления и функции, описывающие 


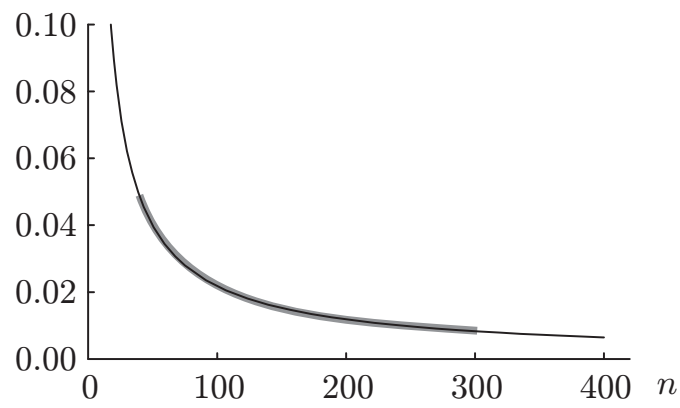

Рис. 5

кривые, имеют тот же вид); как и выше, мы проводили численные вычисления до уровня $n=300$. Результат вычислений представлен на рис. 5 .

Таким образом, предэкспоненциальный фактор (3.14) поднимается в показатель экспоненты. Это завершает получение правильной структуры показателя экспоненты.

В итоге соотношение спуска в духовом секторе имеет вид

$$
\begin{aligned}
{ }_{321}\left\langle V_{3}^{\mathrm{gh}} \mid V_{1}^{\mathrm{gh}}\right\rangle_{1} & =-J_{0} N_{3} \frac{i}{4} \operatorname{det}(1-S X)_{23}\langle+|\left(b_{0}^{2}-b_{0}^{3}\right) e^{-b^{2} S c^{3}-b^{3} S c^{2}}= \\
& =-J_{0} N_{3} \frac{i}{4} \operatorname{det}(1-S X)_{23}\left\langle V_{2}\right| \equiv \widetilde{Z}_{\mathrm{ov}}^{\mathrm{gh}}{ }_{23}\left\langle V_{2}^{\mathrm{gh}}\right| .
\end{aligned}
$$

Здесь мы восстановили опущенные ранее коэффициенты.

3.2.2. "Неоверлэп-вычисления" в секторе духов. Проведем прямое, без использования условия оверлэпа, вычисление соотношения спуска.

Рассмотрим выражение (см. (3.3))

$$
321\left\langle+\left|e^{-b^{r} X^{r s} c^{s}} b_{+}^{1} b_{-}^{1} e^{b^{*} S c^{*}}\right|+\right\rangle_{1} .
$$

Удобно переписать вставку в виде

$$
b_{+}^{1} b_{-}^{1} \equiv A+b_{0}^{1} B
$$

где введены обозначения (см. приложение)

$$
\begin{gathered}
A \equiv b K b L+b K b^{*} K-b L b^{*} L+b^{*} L b^{*} K, \\
B \equiv b T-b^{*} T
\end{gathered}
$$

(временно мы опустим индекс “1”, характеризующий моду первой струны).

Подставим вставку (3.30) в выражение (3.29) и выделим экспоненту $e^{-W_{2}}$, содержащую моду только второй и третьей струны. Получаем

$$
{ }_{32}\langle+| e^{-W_{2}}{ }_{1}\left\langle+\left|e^{-W_{1}-b_{0}^{1} w}\left(A+b_{0} B\right) e^{b^{*} S c^{*}}\right|+\right\rangle_{1} .
$$


Здесь $w, W_{1}$ и $W_{2}$ те же, что и ранее. Разложим экспоненту по нулевой моде $b_{0}^{1}$ (опустим множитель $32\langle+| e^{-W_{2}}$ ):

$$
{ }_{1}\left\langle+\left|e^{-W_{1}}\left(1-b_{0} w\right)\left(A+b_{0} B\right) e^{b^{*} S c^{*}}\right|+\right\rangle_{1}={ }_{1}\left\langle+\left|e^{-W_{1}}\left(A-b_{0} w A+b_{0} B\right) e^{b^{*} S c^{*}}\right|+\right\rangle_{1} .
$$

Первое слагаемое не дает вклада. В оставшихся двух слагаемых пронесем $b_{0} \mathrm{~K}$ левому вакууму:

$$
{ }_{1}\left\langle-\left|e^{-W_{1}} B e^{b^{*} S c^{*}}\right|+\right\rangle_{1}-{ }_{1}\left\langle-\left|e^{-W_{1}} A w e^{b^{*} S c^{*}}\right|+\right\rangle_{1} .
$$

Прямое вычисление величины (3.31) дает

$$
\begin{aligned}
\left\langle V_{3}^{\mathrm{gh}} \mid V_{1}^{\mathrm{gh}}\right\rangle=- & 2 \operatorname{det}\left(Z^{-1}\right)_{23}\langle+| \exp \left\{-\lambda^{b} Z S \lambda^{c}-\sum_{\substack{n=0 \\
m=1}}^{\infty} \sum_{\substack{p, q=2 \\
3}}^{3} b_{n}^{p} X_{n m}^{p q} c_{m}^{q}\right\} \times \\
& \times\left[\lambda^{b} Z T+2 \bar{X} Z L \lambda^{b} Z K-2 \bar{X} Z K \lambda^{b} Z L-2 \lambda^{b} Z L \lambda^{b} Z K \bar{U}^{1 q} c^{q}\right] .
\end{aligned}
$$

В экспоненте получена матрица $U_{n m}^{p q}$ (см. (3.9)), как и в предыдущем случае, и это не удивительно, так как при взятии среднего от двух экспонент предэкспонента не вносит вклада в показатель. Для дальнейших вычислений мы используем численно установленное равенство $\bar{X} Z L=\bar{X} Z K$ (которое выполняется на любом уровне). Учитывая его, получаем

$$
-2 \operatorname{det}\left(Z^{-1}\right)_{23}\langle+| e^{-\check{b}^{p} U^{p q} c^{q}}\left[\theta \check{b}^{r} X^{r 1} Z T+2 \check{b}^{p} X^{p 1} Z L \check{b}^{r} X^{r 1} Z K \bar{U}^{1 q} c^{q}\right],
$$

где $\theta \equiv(1-2 \bar{X} Z L)$ и $\check{b}^{r} \equiv\left(b_{0}^{r}, b_{1}^{r}, b_{2}^{r}, \ldots\right)$.

Подчеркнем, что $\check{b}^{p} X^{p 1}$ и $\check{b}^{p} U^{p q}$ содержат нулевые моды.

Так же, как и при первом способе вычислений, проанализируем структуру получившейся предэкспоненты по $b_{0}^{2,3}$. Слагаемые, не содержащие $b_{0}$, имеют вид

$$
\theta b^{p} \widehat{X}^{p 1} Z T+2 b^{p} \widehat{X}^{p 1} Z L b^{r} \widehat{X}^{r 1} Z K \bar{U}^{1 q} c^{q} .
$$

В масштабе $\mathfrak{J}_{0}$ (см. ниже) они исчезают. На рис. 6 (рис. $6 \mathrm{a}-\theta b^{p} \widehat{X}^{p 1} Z T$, рис. $6 б-$ $\left.2 b^{p} \widehat{X}^{p 1} Z L b^{r} \widehat{X}^{r 1} Z K \bar{U}^{1 q} c^{q}\right)$ отражено поведение этих слагаемых. Они стремятся к нулю при $n \rightarrow \infty$ как $\sim 0.856 / n^{0.819}$ и $\sim 0.073 / n^{0.142}$, соответственно.

Рассмотрим слагаемые, пропорциональные $b_{0}^{2}$ и $b_{0}^{3}$ :

$$
\begin{aligned}
-\theta b_{0}^{2} \bar{X}^{21} Z T-\theta b_{0}^{3} \bar{X}^{31} Z T- \\
\quad-2\left(b_{0}^{2} \bar{X}^{21} Z L b^{p} \widehat{X}^{p 1} Z K+b_{0}^{3} \bar{X}^{31} Z L b^{p} \widehat{X}^{p 1} Z K\right) \bar{U}^{1 q} c^{q}+ \\
\quad+2\left(b_{0}^{2} \bar{X}^{21} Z K b^{p} \widehat{X}^{p 1} Z L+b_{0}^{3} \bar{X}^{31} Z K b^{p} \widehat{X}^{p 1} Z L\right) \bar{U}^{1 q} c^{q} \equiv \\
\equiv b_{0}^{2}\left(\mathfrak{J}_{0}+b^{2} \overline{\mathfrak{A}}^{2} \bar{U}^{1 q} c^{q}+b^{3} \overline{\mathfrak{A}}^{3} \bar{U}^{1 q} c^{q}\right)+b_{0}^{3}\left(\mathfrak{J}_{0}^{\prime}+b^{2} \overline{\mathfrak{B}}^{2} \bar{U}^{1 q} c^{q}+b^{3} \overline{\mathfrak{B}}^{3} \bar{U}^{1 q} c^{q}\right) ;
\end{aligned}
$$

здесь мы ввели обозначения

$$
\begin{gathered}
\mathfrak{J}_{0}=-\theta \bar{X}^{21} Z T, \quad \mathfrak{J}_{0}^{\prime}=-\theta \bar{X}^{31} Z T, \\
\overline{\mathfrak{A}}^{i}=-2\left(\widehat{X}^{i 1} Z K \bar{X}^{21} Z L-\widehat{X}^{i 1} Z L \bar{X}^{21} Z K\right), \quad i=2,3, \\
\overline{\mathfrak{B}}^{i}=-2\left(\widehat{X}^{i 1} Z K \bar{X}^{31} Z L-\widehat{X}^{i 1} Z L \bar{X}^{31} Z K\right) .
\end{gathered}
$$



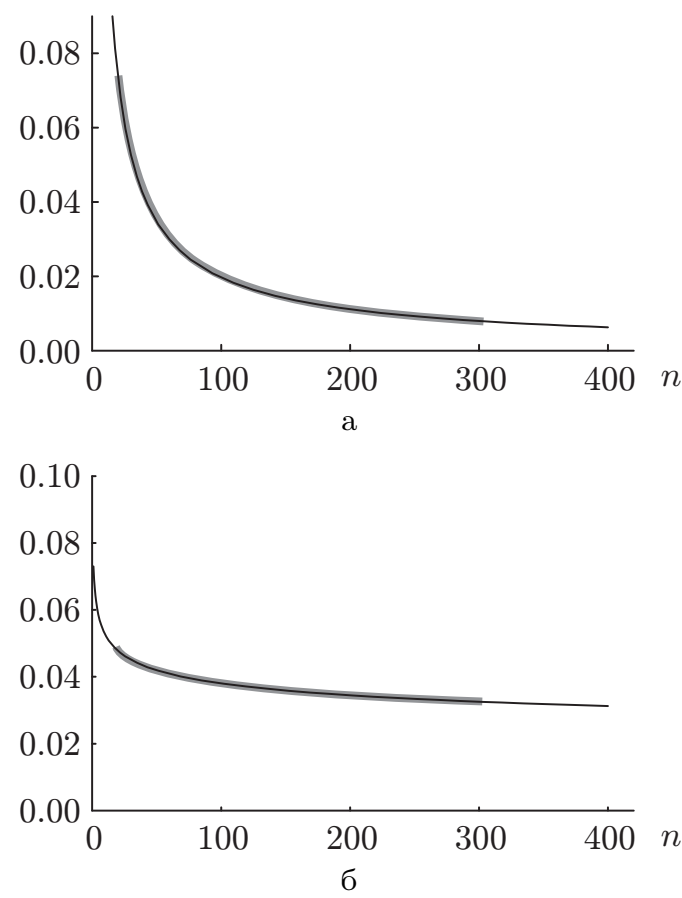

Рис. 6

Для правильной структуры вакуумов необходимо, но не достаточно, чтобы выполненялось условие $\mathfrak{J}_{0}^{\prime}+\mathfrak{J}_{0} \rightarrow 0$. Численные вычисления показывают, что $\mathfrak{J}_{0}^{\prime}+\mathfrak{J}_{0}=0$ начиная с первого уровня.

Квадратичное по $b_{0}$ слагаемое в предэкспоненте имеет вид

$$
2 b_{0}^{2} b_{0}^{3}\left(-\bar{X}^{21} Z L \bar{X}^{31} Z K+\bar{X}^{31} Z L \bar{X}^{21} Z K\right) \bar{U}^{1 q} c^{q} .
$$

Следующим шагом, как и в предыдущем вычислении, является поднятие линейных и квадратичных по $b_{0}$ слагаемых из предэкспоненты в показатель экспоненты. Выполняя это, мы опять получаем некоторые новые матрицы $V^{p q}, p=2,3$. Снова запишем систему уравнений для выполнения условий (3.23) и (3.24):

$$
\begin{gathered}
\mathfrak{J}_{0}\left(\bar{V}^{2 q}+\bar{V}^{3 q}\right)=2\left(\bar{X}^{21} Z L \bar{X}^{31} Z K-\bar{X}^{31} Z L \bar{X}^{21} Z K\right) \bar{U}^{1 q}, \\
\left(\bar{V}^{2 q}+\bar{V}^{3 q}\right) c^{q} b^{p} \widehat{V}^{p s} c^{s}=0, \\
\widehat{V}^{p q}=\frac{\overline{\mathfrak{A}}^{p}}{\mathfrak{J}_{0}} \otimes \bar{U}^{1 q}, \\
\widehat{V}^{p q}=\frac{\overline{\mathfrak{B}}^{p}}{\mathfrak{J}_{0}} \otimes \bar{U}^{1 q} .
\end{gathered}
$$

Эта система похожа на систему (3.25), однако она написана через матрицы $X$, а не через матрицы $U$. 
Условие совместности этой системы имеет вид

$$
\frac{\overline{\mathfrak{A}}^{r}}{\mathfrak{J}_{0}}+\frac{\overline{\mathfrak{B}}^{r}}{\mathfrak{J}_{0}} \rightarrow 0, \quad r=2,3 .
$$

Полученная численно зависимость этого условия от номера уровня показана на рис. 7 и описывается функцией $\sim 0.705 / n^{0.783}$. Видно, что она стремится к нулю при $n \rightarrow \infty$.

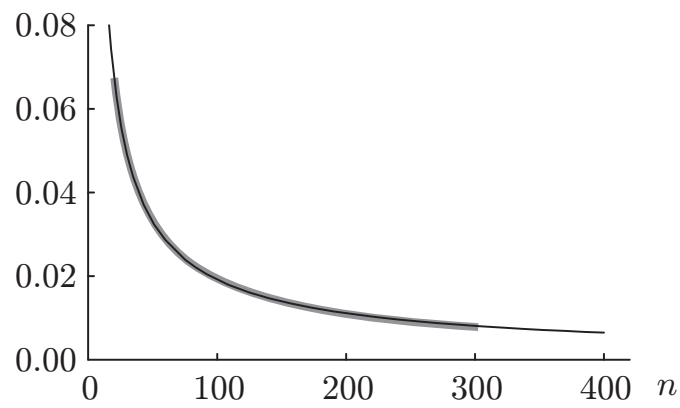

Рис. 7

Для решения системы (3.36) мы используем тот же метод, что и в п. 3.2.1.

Отметим, что система (3.36) определяет только сумму строк $\bar{V}^{2 q}+\bar{V}^{3 q}$. Положив $\bar{U}^{p q}=-\bar{V}^{p q}$, удовлетворим уравнению (3.23). Из этого следует, что уравнение (3.36а) можно переписать как

$$
\mathfrak{J}_{0}\left(\bar{U}^{2 q}+\bar{U}^{3 q}\right)=-2\left(\bar{X}^{21} Z L \bar{X}^{31} Z K-\bar{X}^{31} Z L \bar{X}^{21} Z K\right) \bar{U}^{1 s} .
$$

Используя $\bar{U}^{1 q}=-\left(\bar{U}^{2 q}+\bar{U}^{3 q}\right)$, перепишем это равенство в виде

$$
\mathfrak{J}_{0}=2\left(\bar{X}^{21} Z L \bar{X}^{31} Z K-\bar{X}^{31} Z L \bar{X}^{21} Z K\right)
$$

или, раскрыв обозначения,

$$
-(1-2 \bar{X} Z L) \bar{X}^{21} Z(L-K)=2\left(\bar{X}^{21} Z L \bar{X}^{31} Z K-\bar{X}^{31} Z L \bar{X}^{21} Z K\right) .
$$

На рис. 8 показано поведение этого выражения, переписанного как

$$
1-2 \frac{\bar{X}^{21} Z L \bar{X}^{31} Z K-\bar{X}^{31} Z L \bar{X}^{21} Z K}{\mathfrak{J}_{0}},
$$

при $n \rightarrow \infty$. Функция, описывающая эту кривую, имеет вид $\sim 1.13 / n^{0.86}$.

Уравнение (3.36в) определяет матрицу $\widehat{V}^{p q}$, которая должна удовлетворять условиям (3.24). Численные вычисления показывают, что второе из этих условий имеет такое же поведение, как и первое. Таким образом, мы приведем результат вычислений только для первого из этих условий. Мы вычисляем модуль максимального элемента $\operatorname{Max}\left|\widehat{U}^{22}+\widehat{V}^{22}\right|$ для $n=40, \ldots, 300$, отбрасывая при этом последние 20 строк и столбцов в матрицах, участвующих в вычислениях. 


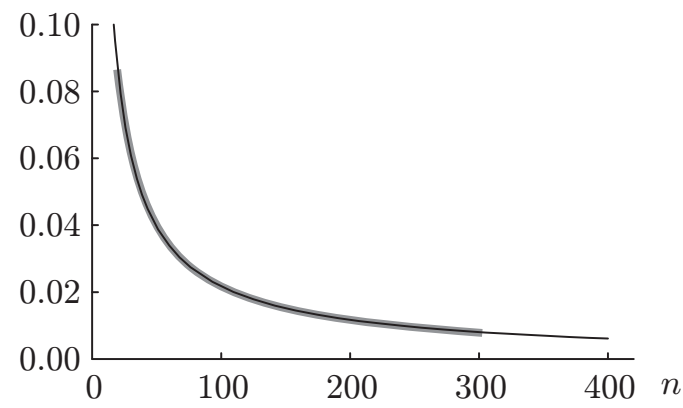

Рис. 8

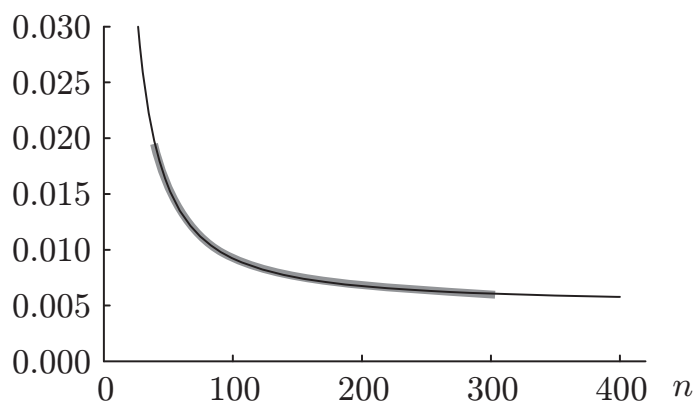

Рис. 9

Величина $\operatorname{Max}\left|\widehat{U}^{22}+\widehat{V}^{22}\right|$ описывается функцией $\sim 0.005+2.17 / n^{1.36}$ (рис. 9).

Окончательный результат при вычислении соотношения спуска в секторе духов имеет вид

$$
\begin{aligned}
{ }_{321}\left\langle V_{3}^{\mathrm{gh}} \mid V_{1}^{\mathrm{gh}}\right\rangle_{1} & =\mathfrak{J}_{0} N_{3} \frac{i}{2} \operatorname{det}(1-S X)_{23}\langle+|\left(b_{0}^{2}-b_{0}^{3}\right) e^{-b^{2} S c^{3}-b^{3} S c^{2}}= \\
& =\mathfrak{J}_{0} N_{3} \frac{i}{2} \operatorname{det}(1-S X)_{23}\left\langle V_{2}^{\mathrm{gh}}\right| \equiv \widetilde{Z}_{\mathrm{nov} 23}^{\mathrm{gh}}\left\langle V_{2}^{\mathrm{gh}}\right| .
\end{aligned}
$$

Здесь мы восстановили коэффициенты, опущенные ранее.

3.2.3. Учет сектора материи. Нормировка $\widetilde{Z}_{3}$ в соотношении спуска $\left\langle V_{3} \mid V_{1}\right\rangle$ содержит два множителя: нормировку в секторе духов $\widetilde{Z}^{\text {gh }}$ и нормировку в секторе материи $\widetilde{Z}^{\mathrm{m}}$, а именно $\widetilde{Z}_{3}=\widetilde{Z}^{\mathrm{m}} \widetilde{Z}^{\mathrm{gh}}$. В секторе духов с использованием условия оверлэпа нормировка имеет вид

$$
\widetilde{Z}_{\mathrm{ov}}^{\mathrm{gh}}=-\frac{i}{4} N_{3} J_{0} \operatorname{det}(1-S X),
$$

при использовании способа без условия оверлэпа -

$$
\widetilde{Z}_{\text {nov }}^{\text {gh }}=\frac{i}{2} N_{3} \mathfrak{J}_{0} \operatorname{det}(1-S X) .
$$

В секторе материи нормировочный множитель представи́м в виде

$$
\widetilde{Z}^{\mathrm{m}}=\operatorname{det}\left(1+V^{11 \mathrm{~m}} S\right)^{-13},
$$


где $V^{11 \mathrm{~m}}$ - матрица Неймана для сектора материи [2], [9]. Результат для сектора материи был получен в работе [13] и проверен нами. Так как вычисления в секторе материи проводятся довольно однозначно, мы приводим только ответ без промежуточных вычислений.

Численные вычисления полного нормировочного множителя с учетом секторов материи и духов дают в рассматриваемых системах два разных результата, которые показаны на рис. 10а, б.
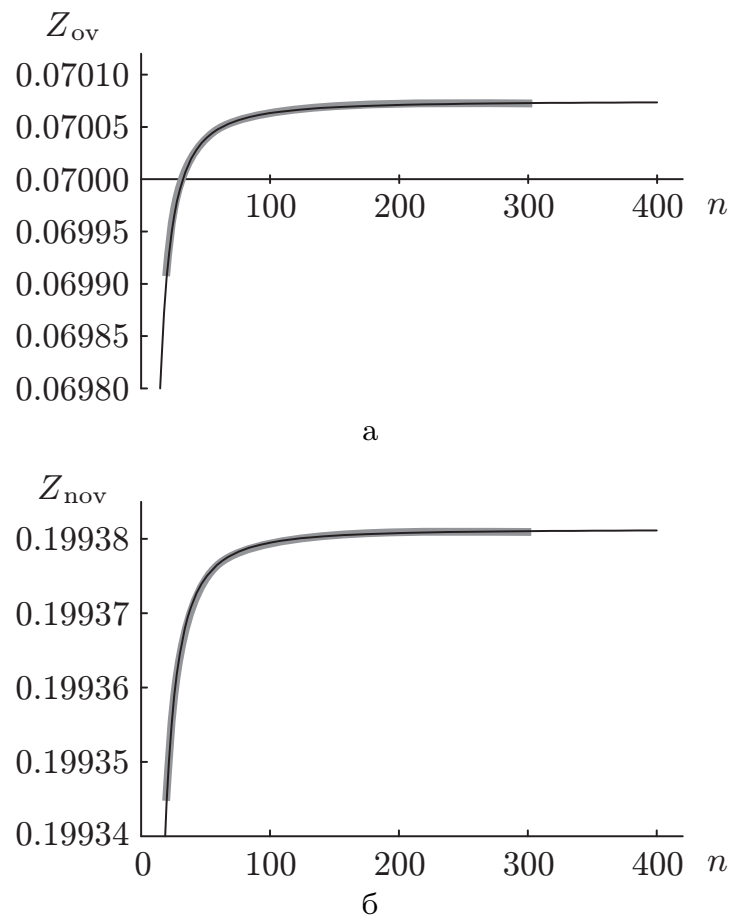

Рис. 10

Поведение этих кривых описывается функциями $\widetilde{Z}_{3} \sim 0.070075-0.024 / n^{1.666}$ и $\widetilde{Z}_{3} \sim 0.199381-0.01 / n^{1.87}$, соответственно.

В итоге мы получили соотношение спуска $\left\langle V_{3} \mid V_{1}\right\rangle$, используя две различные схемы вычислений. В обоих случаях использовался осцилляторный метод обрезания по уровням с различной обработкой вставки в средней точке. Обе схемы вычисления дали вершину $\left\langle V_{2}\right|$ с двумя различными нормировочными множителями. Впервые трудности в использовании метода обрезания по уровням осциллятора были отмечены в работе [21].

\section{ПРИЛОЖЕНИЕ}

В настоящей статье мы использовали следующие обозначения для вакуума [2] в секторе материи:

$$
a_{n}|0\rangle=0, \quad n \geqslant 0, \quad\langle 0 \mid 0\rangle=1 ;
$$


в секторе духов:

$c_{n}|+\rangle=0, \quad n \geqslant 0, \quad b_{m}|+\rangle=0, \quad m \geqslant 1, \quad|-\rangle=b_{0}|+\rangle, \quad\langle-\mid+\rangle=\langle+\mid-\rangle=1$.

Вершина $\left\langle V_{3}\right|$ имеет вид

$$
\left\langle V_{3}\right|={ }_{321}\langle+| \exp \left\{-\sum_{r, s=1}^{3} \sum_{\substack{n=0 \\ m=1}} b_{n}^{r} X_{n m}^{r s} c_{m}^{s}\right\} N_{3}
$$

где

$$
{ }_{321}\langle+| \equiv{ }_{3}\left\langle+{ }_{2}\left\langle+{ }_{1}\langle+| .\right.\right.
$$

Матрицы Неймана обладают следующими свойствами [2], [19]:

$$
\begin{gathered}
X_{n m}^{r, r}+X_{n m}^{r, r+1}+X_{n m}^{r, r-1}=S_{n m}, \quad n \neq 0, \\
X_{0 m}^{r, r}+X_{0 m}^{r, r+1}+X_{0 m}^{r, r-1}=0,
\end{gathered}
$$

где $X_{n m}^{r, r+2}=X_{n m}^{r, r-1} ;$ при фиксированном втором индексе эти свойства сохраняются.

Вставку $b_{+} b_{-}$с выделенной нулевой модой удобно переписать в виде

$b_{+} \equiv b\left(\frac{\pi}{2}\right)=\sum_{-\infty}^{\infty} i^{n} b_{n}=b K+b_{0}+b^{*} L, \quad b_{-} \equiv b\left(-\frac{\pi}{2}\right)=\sum_{-\infty}^{\infty} i^{-n} b_{n}=b L+b_{0}+b^{*} K$,

где

$$
\begin{gathered}
b^{j} \equiv\left(b_{1}^{j}, b_{2}^{j}, \ldots\right), \quad b^{j *} \equiv\left(b_{-1}^{j}, b_{-2}^{j}, \ldots\right), \\
c^{j *} \equiv\left(c_{-1}^{j}, c_{-2}^{j}, \ldots\right)^{\mathrm{T}}, \quad c^{j} \equiv\left(c_{1}^{j}, c_{2}^{j}, \ldots\right)^{\mathrm{T}}, \\
K \equiv(i,-1,-i, 1, \ldots)^{\mathrm{T}}, \quad L \equiv(-i,-1, i, 1, \ldots)^{\mathrm{T}}, \\
T \equiv L-K=(-2 i, 0,2 i, 0, \ldots)^{\mathrm{T}} .
\end{gathered}
$$

В показателе экспоненты вершины $V_{3}$ величина, содержащая моды первой струны, имеет вид

$$
\begin{aligned}
W_{1} & =\sum_{n, m=1}^{\infty} b_{n}^{1} X_{n m}^{11} c_{m}^{1}+\sum_{s=2}^{3} \sum_{n, m=1}^{\infty} b_{n}^{1} X_{n m}^{1 s} c_{m}^{s}+\sum_{r=2}^{3} \sum_{\substack{n=0 \\
m=1}}^{\infty} b_{n}^{r} X_{n m}^{r 1} c_{m}^{1} \equiv \\
& \equiv b^{1} \widehat{X} c^{1}+b^{1} \lambda^{c}+\lambda^{b} c^{1}
\end{aligned}
$$

где

$$
\begin{gathered}
\lambda^{c} \equiv \sum_{s=2}^{3} \widehat{X}^{1 s} c^{s}, \quad \lambda^{b} \equiv \sum_{r=2}^{3}\left(b_{0}^{r} \bar{X}^{r 1}+b^{r} \widehat{X}^{r 1}\right), \\
W_{2}=\sum_{p, q=2}^{3} \sum_{n, m=1}^{\infty} b_{n}^{p} X_{n m}^{p q} c_{m}^{q} .
\end{gathered}
$$


Благодарности. Авторы благодарны Д. М. Белову и А. С. Кошелеву за полезное обсуждение, а также Е. Фуксу и М. Кройтеру за переписку и полезные замечания. Работа была частично поддержана РФФИ (грант № 05-01-00758) и Программой поддержки ведущих научных школ (грант № НШ-2052.2003.1). Работа И. Я. Арефьевой частично поддержана INTAS (грант № 03-51-6346).

\section{Список литературы}

[1] E. Witten, Nucl. Phys. B, 268 (1986), 253.

[2] D. Gross, A. Jevicki, Nucl. Phys. B, 283 (1987), 1; 287 (1987), 225; 293 (1987), 29.

[3] A. LeClair, M. Peskin, C. Preitschopf, Nucl. Phys. B, 317 (1989), 411; 464.

[4] K. Ohmori, A review on tachyon condensation in open string field theories, hep-th/0102085; I. Ya. Aref'eva, D. M. Belov, A. A. Giryavets, A. S. Koshelev, P. B. Medvedev, Noncommutative field theories and (super) string field theories, hep-th/0111208.

[5] W. Taylor, B. Zwiebach, D-branes, tachyons, and string field theory, hep-th/0311017.

[6] E. Cremmer, A. Schwimmer, C. B. Thorn, Phys. Lett. B, 179 (1986), 57.

[7] S. Samuel, Phys. Lett. B, 181 (1986), 255.

[8] N. Ohta, Phys. Rev. D, 34 (1986), 3785; 35 (1987), 2627 (E).

[9] W. Taylor, Perturbative diagrams in string field theory, hep-th/0207132.

[10] V. A. Kostelecky, S. Samuel, Nucl. Phys. B, 336 (1990), 263.

[11] N. Moeller, W. Taylor, Nucl. Phys. B, 583 (2000), 105; hep-th/0002237.

[12] A. Sen, B. Zwiebach, JHEP, 03 (2000), 002; hep-th/9912249; L. Rastelli, A. Sen, B. Zwiebach, Adv. Theor. Math. Phys., 5 (2002), 393; hep-th/0102112; I. Ya. Aref'eva, D. M. Belov, A. S. Koshelev, P. B. Medvedev, Nucl. Phys. B, 638 (2002), 3; hep-th/0011117; 21; hep-th/0107197; K. Ohmori, Level-expansion analysis in NS superstring field theory revisited, hep-th/0305103.

[13] E. Fuchs, M. Kroyter, JHEP, 12 (2005), 031; hep-th/0508010.

[14] D. M. Belov, A. Konechny, JHEP, 10 (2002), 049; hep-th/0207174; Phys. Lett. B, 558 (2003), 111; hep-th/0210169; E. Fuchs, M. Kroyter, A. Marcus, JHEP, 11 (2002), 046; hep-th/0210155; 11 (2003), 039; hep-th/0307148.

[15] D. M. Belov, Phys. Rev. D, 69 (2004), 126001; hep-th/0308147.

[16] L. Rastelli, A. Sen, B. Zwiebach, JHEP, 03 (2002), 029; hep-th/0111281.

[17] I. Y. Aref'eva, P. B. Medvedev, A.P. Zubarev, Phys. Lett. B, 240 (1990), 356; Nucl. Phys. B, 341 (1990), 464; C. R. Preitschopf, C. B. Thorn, S. A. Yost, Nucl. Phys. B, 337 (1990), 363.

[18] S. Samuel, Nucl. Phys. B, 296 (1988), 187.

[19] V. A. Kostelecky, R. Potting, Phys. Rev. D, 63 (2001), 046007; hep-th/0008252.

[20] I. Kishimoto, JHEP, 12 (2001), 007; hep-th/0110124.

[21] K. Okuyama, JHEP, 03 (2002), 050; hep-th/0201136.

Поступила в редакцию 16.06.2006 